\title{
ReEValuación de los bosques tropicales estacionalmente seCos de la Reserva de la Biosfera Sierra del Abra Tanchipa y áreas con potencial para su conservación Reevaluation of the seasonally dry tropical forest of the Biosphere Reserve Sierra DEL ABRA AND SUITABLE AREAS FOR CONSERVATION
}

\author{
- Romina Gutiérrez-Hernández ${ }^{1}$, Prancisco Javier Sahagún-Sánchez², Pablo Delgado-Sánchez 2 ,

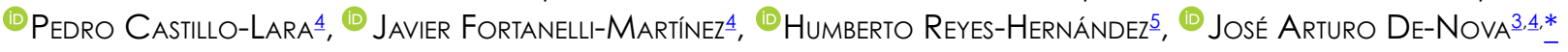

\author{
' Programa de Maestría en Ciencias Agropecuarias, Facultad de Agronomía y Veterinaria, Universidad Autónoma de San Luis \\ Potosí, San Luis Potosí, México. \\ 2 Departamento de Políticas Públicas, Centro Universitario de Ciencias Económico Administrativas, Universidad de Guadalajara, \\ Zapopan, Jalisco, México. \\ ${ }^{3}$ Facultad de Agronomía y Veterinaria, Universidad Autónoma de San Luis Potosí, San Luis Potosí, México. \\ ${ }^{4}$ Instituto de Investigación de Zonas Desérticas, Universidad Autónoma de San Luis Potosí, San Luis Potosí, México. \\ ${ }^{5}$ Facultad de Ciencias Sociales y Humanidades, Universidad Autónoma de San Luis Potosí, San Luis Potosí, México. \\ *Autor para la correspondencia: arturo.denova@gmail.com
}

\section{Resumen}

Antecedentes: La Reserva de la Biosfera Sierra del Abra Tanchipa incluye bosques tropicales estacionalmente secos, considerados como ecosistemas vulnerables en el Neotrópico. Recientemente se elaboró una cartografía sobre su distribución que requería una verificación.

Pregunta: ¿Cuál es la distribución espacial de los bosques tropicales estacionalmente secos de la Reserva en la actualidad? ¿Cuáles sitios dentro del área de influencia pueden ser propuestos como áreas destinadas voluntariamente para la conservación?

Sitio de estudio y fechas: Reserva de la Biosfera Sierra del Abra Tanchipa, San Luis Potosí, México, de agosto de 2014 a junio de 2019.

Métodos: Se estudiaron 17 sitios de muestreo, elegidos completamente al azar y se estimó el índice de valor de importancia relativa. Se realizó un análisis de similitud para reclasificar las variantes de vegetación. Se revisó la cartografía existente de vegetación, se actualizó su distribución espacial y se identificaron áreas potenciales para la conservación.

Resultados: Se registraron 177 especies de plantas leñosas, de las cuales154 son arbóreas o arbustivas y 23 trepadoras. Las familias más ricas fueron Fabaceae, Euphorbiaceae, Apocynaceae y Malpighiaceae. Se reconocieron cuatro variantes de vegetación: selva baja caducifolia, selva baja subcaducifolia, selva mediana subcaducifolia y selva mediana subperennifolia. Se identificaron cinco áreas en la zona de influencia de la Reserva que podrían ser destinadas voluntariamente a la conservación.

Conclusiones: La correcta delimitación espacial de las cubiertas de vegetación de la Reserva es útil para promover acciones de manejo y conservación que garanticen la permanencia de los bosques tropicales estacionalmente secos.

Palabras clave: Bosque tropical caducifolio, cartografía, cubierta vegetal, índice del valor de importancia.

\section{Abstract}

Background: The Sierra del Abra Tanchipa Biosphere Reserve includes seasonally dry tropical forests, considered vulnerable ecosystems in the Neotropics. Verification of the recently elaborated cartography of its distribution was needed.

Question: What is the current spatial distribution of the seasonally dry tropical forest variants in the Reserve? What sites inside its influence area could be voluntarily dedicated to conservation?

Study site and research period: Sierra del Abra Tanchipa Biosphere Reserve, San Luis Potosi, Mexico, between August 2014 to June 2019.

Methods: Seventeen completely random rectangular sampling sites were studied, and the relatively important value index was estimated. A similarity analysis was performed to reclassify vegetation variants. The existing cartography of vegetation was revised, their spatial distribution was updated, and potential areas suitable to conservation were identified.

Results: We recorded 177 species of woody plants, of which 154 are arboreous or shrubby and 23 climbing. The richest families were Fabaceae, Euphorbiaceae, Apocynaceae, and Malpighiaceae. Four vegetation variants were recognized: selva baja caducifolia, selva baja subcaducifolia, selva mediana subcaducifolia y selva mediana subperennifolia. Five areas in the buffer zone were identified suitable to be voluntarily dedicated to conservation.

Conclusions: The correct spatial delimitation of the cover of vegetation of the Reserve are useful to promote management and conservation actions that ensure the permanence of the reserve's seasonally dry tropical forests.

Keywords: Cartography, deciduous tropical forest, importance value index, vegetation cover.

Este artículo se encuentra bajo los términos de la licencia Creative Commons Attribution License CCBY-NC (4.0) internacional. https://creativecommons.org/licenses/by-nc/4.0/ 
A nivel mundial, los bosques tropicales estacionalmente secos (BTES) representan el $42 \%$ de los ecosistemas tropicales, y tienen una amplia distribución en América (Milles et al. 2006, Ceballos et al. 2010, Espinosa et al. 2012). Estas comunidades vegetales se han clasificado de distintas maneras como: bosque tropical deciduo, bosque tropical caducifolio y subcaducifolio, selva baja caducifolia y subcaducifolia, selva mediana caducifolia y subcaducifolia (Miranda \& Hernández-X 1963, Rzedowski 1978, Challenger \& Soberón 2008, INEGI 2009, Rzedowski \& Calderón de Rzedowski 2013). Los BTES son considerados como una comunidad densa dominada por árboles de tamaño bajo a mediano (Becerra 2005) que se distinguen por recibir alrededor del $80 \%$ de la precipitación durante cuatro meses al año, con una media de lluvia que puede sobrepasar los $200 \mathrm{~mm}$ por mes, en contraste, el período de sequía suele durar entre cinco a seis meses al año (Maass \& Burgos 2011, Espinosa et al. 2012). Así, una de las características más conspicuas de los BTES es la fenología distintiva de la mayoría de las plantas, ligada a la pérdida estacional de las hojas y del bosque en general, con una época sin hojas durante la estación seca y una fisonomía de bosque siempreverde a lo largo de la estación lluviosa (Espinosa et al. 2012). Los BTES en México se extienden desde la vertiente del Pacífico, con algunas interrupciones en las porciones más húmedas de Nayarit y Oaxaca, en manchones discontinuos sobre la vertiente del Golfo de México y la península de Yucatán y penetran al continente a través de cuencas como las de los ríos Santiago y Balsas (Trejo 2005, De Nova et al. 2012). Además, su distribución en México se considera el bastión más norteño en el continente americano (Trejo 2010).

Otra característica de estos bosques es que contribuyen con elementos primordiales para la riqueza biológica del territorio mexicano, por su alta diversidad y su elevado nivel de endemismo (Ceballos \& García 1995, Lott \& Atkinson 2002, Trejo \& Dirzo 2002, Ceballos et al. 2010, Sosa \& De-Nova 2012, Meave et al. 2012, Rzedowski \& Calderón de Rzedowski 2013, Sosa et al. 2018). En general, la flora de México es considerada a nivel mundial como una de las más ricas en especies con alrededor de 23,314 especies nativas y 11,600 especies endémicas, de las cuales al menos 3,225 habitan en los BTES (Rzedowski \& Calderón de Rzedowski 2013, Villaseñor 2016). Es considerado como uno de los ecosistemas más vulnerables y en peligro de extinción del país y del mundo, debido a actividades antropogénicas, tales como la deforestación y la fragmentación de los ecosistemas (Pennington et al. 2000, Brooks et al. 2004, Dick \& Wright 2005, Ceballos et al.
2010). En la región de la Huasteca potosina, los BTES han sido afectados por cambios en los usos de la tierra, donde resalta la deforestación causada por el proyecto de riego Pujal-Coy en la década de 1970 (Reyes-Hernández et al. 2006). Por otro lado, se ha registrado el aumento del cultivo de caña de azúcar hacia tierras marginales (AguilarRivera et al. 2010) y otras actividades como la ganadería y la extracción selectiva de especies leñosas, lo cual afecta a su riqueza y diversidad de especies (Alanís-Rodríguez et al. 2010). Debido a estas actividades la selva baja caducifolia es la que presenta mayor superficie deforestada, seguida de la selva baja subcaducifolia para la región (Reyes-Hernández et al. 2018).

En México, una de las estrategias para mitigar el deterioro de los ecosistemas y su biodiversidad, además de propiciar su conservación, ha sido la creación de las Áreas Naturales Protegidas (ANP). Actualmente existen 182 ANP en la República Mexicana a las que se suman 354 áreas de conservación en la modalidad de Áreas Destinadas Voluntariamente a la Conservación (ADVC) (CONANP 2018a, b, SEMARNAT-CONANP 2016, Silva-Aparicio et al. 2018, CONANP 2020). Si bien en la última década se han decretado nuevas ANP que han hecho posible el incremento de estos ecosistemas con una representatividad del $7 \%(1,658,419$ ha) de la superficie total de las ANP (CONANP 2018a), los BTES son los ecosistemas menos representados y cubren alrededor del $1 \%$ del territorio nacional (Ordoñez \& Flores 1995, Ceballos et al. 2010).

En San Luis Potosí, la Reserva de la Biosfera Sierra del Abra Tanchipa (RBSAT), es reconocida como Región Terrestre Prioritaria para la Conservación por su alta biodiversidad (Arriaga-Cabrera et al. 2009, Reyes-Hernández et al. 2018). Esta ANP se encuentra inmersa en la Sierra Madre Oriental (SMOr) y es considerada importante desde la perspectiva biogeográfica, por su función como corredor entre la biota Neártica y Neotropical (De-Nova et al. 2018). La riqueza florística de esta ANP está representada por 427 especies de plantas vasculares pertenecientes a 305 géneros y 89 familias; de las cuales, 214 especies corresponden a hierbas, 78 son árboles, 59 son arbustos, 47 son trepadoras, y 29 son epífitas, de acuerdo con su forma de crecimiento (De-Nova et al. 2019). Con respecto a la clasificación de los BTES en la RBSAT, Reyes-Hernández et al. (2018) propusieron la presencia de cuatro variantes de vegetación a partir de la interpretación y análisis de imágenes satelitales SPOT del año 2016, mediante el método de clasificación supervisada en el software ENVI 4.8 (Mas 2005, Lillesand et al. 2004, Leija-Loredo et al. 
2020); sin embargo, en el análisis de la información cartográfica y de campo se encontraron inconsistencias por lo que una reclasificación y delimitación espacial de la distribución de las cubiertas de vegetación en la zona es necesaria. El objetivo del presente estudio fue analizar las variantes de vegetación en los BTES en la Reserva de la Biosfera Sierra del Abra Tanchipa para determinar su distribución actual e identificar sitios con potencial para ser propuestos como áreas destinadas voluntariamente a la conservación de la zona de influencia del ANP.

\section{Materiales y métodos}

Área de estudio. La Reserva de la Biosfera Sierra del Abra Tanchipa (RBSAT) se localiza en los municipios de Ciudad Valles y Tamuín al este del estado de San Luis Potosí. Se ubica entre las coordenadas $22^{\circ} 05^{\prime} 00^{\prime}$ " y $22^{\circ} 24^{\prime} 22^{\prime \prime}$ de latitud norte y $98^{\circ} 52^{\prime} 46^{\prime \prime}$ y $99^{\circ} 01^{\prime} 00^{\prime \prime}$ de longitud oeste, con elevaciones que fluctúan entre los 100 a $820 \mathrm{~m}$ snm y comprende una superficie de 21,464 ha de las cuales 16,758 ha constituyen la zona núcleo, 4,223 ha están destinadas al aprovechamiento sustentable y 482 ha a los usos tradicionales (Figura 1; DOF 1994, CONANP 2014). De acuerdo con el sistema de clasificación de Köppen modificado por García (2004) la RBSAT se caracteriza por un clima cálido subhúmedo $\mathrm{Aw}_{2}$ con lluvias en verano, precipitación pluvial media anual oscila entre los 1,200 y $1,500 \mathrm{~mm}$, con mayores precipitaciones entre junio y octubre y menos de $60 \mathrm{~mm}$ en la temporada seca. La temperatura promedio anual es de $24.5^{\circ} \mathrm{C}$ y la temperatura del mes más frío es menor a los $18{ }^{\circ} \mathrm{C}$ (INEGI 2002, García 2004, Durán 2018). La RBSAT forma parte de la región hidrológica Pánuco (RH26), en la cuenca del río Tamuín y las subcuencas de los ríos Puerco y Valles al Oeste de la sierra y de la subcuenca del río Tampaón al Este. El suelo que predomina en la RBSAT es el leptosol, con textura media y permeabilidad que varía de alta a media, de acuerdo con su contenido de materia orgánica y de arcilla, con una profundidad limitada, de menos de $25 \mathrm{~cm}$ (INEGI

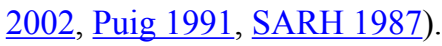

Análisis de la estructura y composición de las variantes de vegetación. Para el muestreo de la vegetación se eligieron sitios al azar en áreas que representaran las distintas variantes determinadas previamente (Reyes-Hernández et al. 2018), con condiciones adecuadas para el acceso y trabajo en campo. Se analizaron 17 sitios de muestreo rectangulares de $100 \mathrm{~m}$ de longitud por $2 \mathrm{~m}$ de anchura $\left(200 \mathrm{~m}^{2}\right)$ ubicados dentro del polígono del ANP. Se re- gistraron y midieron todos los individuos con diámetro a la altura de pecho (DAP) $>2.5 \mathrm{~cm}$, de acuerdo con la metodología sugerida por Gentry (1988) para la evaluación de la vegetación leñosa en selvas tropicales. Para cada individuo se registraron las variables dasométricas de altura y DAP (Ugalde 1981, Castillo-Gómez 2015). Para medir la altura de cada individuo se empleó un dendrómetro Pistola $\operatorname{Haga}{ }^{\circledR}$, para el diámetro de árboles se utilizó una cinta diamétrica y para la delimitación de cada sitio una cinta métrica de $50 \mathrm{~m}$. Con los datos obtenidos en campo se estimó el índice de valor de importancia relativa (VIR) para cada especie, el cual consiste en la sumatoria de los valores relativos de la densidad (densidad absoluta por cada especie/densidad absoluta de todas las especies) $\times 100$, dominancia (dominancia absoluta por especie/dominancia absoluta de todas las especies) $\times 100$ y la frecuencia (frecuencia absoluta por cada especie/frecuencia absoluta de todas las especies) $\times 100$. Se utilizó el análisis de agrupamiento con datos del VIR de las especies y se eligió el índice de similitud de Jaccard como la medida de semejanza entre grupos (sitios de muestreo), con el método de Ward (Ward 1963, Calderón \& Moreno 2019). Se utilizó la función "vegdist" del paquete vegan (Oksanen et al. 2019) para R v.3.6.3 (․ Core Team 2020).

Riqueza de especies y diversidad beta. Para determinar la riqueza total de especies leñosas en el área de estudio se revisaron todos los especímenes depositados en el Herbario Isidro Palacios (SLPM), además del inventario florístico más actual de la RBSAT (De Nova et al. 2019) y con el cual se estandarizó la nomenclatura. Se calculó el índice de biodiversidad taxonómica (IB) propuesto por Squeo et al. (1998), de acuerdo con la siguiente fórmula: $\mathrm{IB}=\mathrm{S} / \mathrm{LnA}$, donde $\mathrm{S}=$ número de especies registradas y $\mathrm{LnA}=\operatorname{loga}$ ritmo natural del tamaño del área. Para determinar el grado de cambio o reemplazo de especies (diversidad beta) entre las diferentes variantes de vegetación del sitio de estudio se realizó un análisis de agrupamiento el cual se estimó con los valores del promedio del VIR por especie y por variante de vegetación mediante el índice de Wilson y Shmida ( $\underline{A n}-$ derson et al. 2011), para este análisis se utilizó el programa de cálculo PAST versión 3.1 (Hammer et al. 2001).

Distribución de las variantes de vegetación e identificación de áreas con potencial para la conservación. Se utilizó la información obtenida del análisis de agrupación de las variantes de vegetación para proceder a la reclasificación del mapa de cobertura de vegetación y usos de suelo elaborado previamente por Reyes-Hernández 

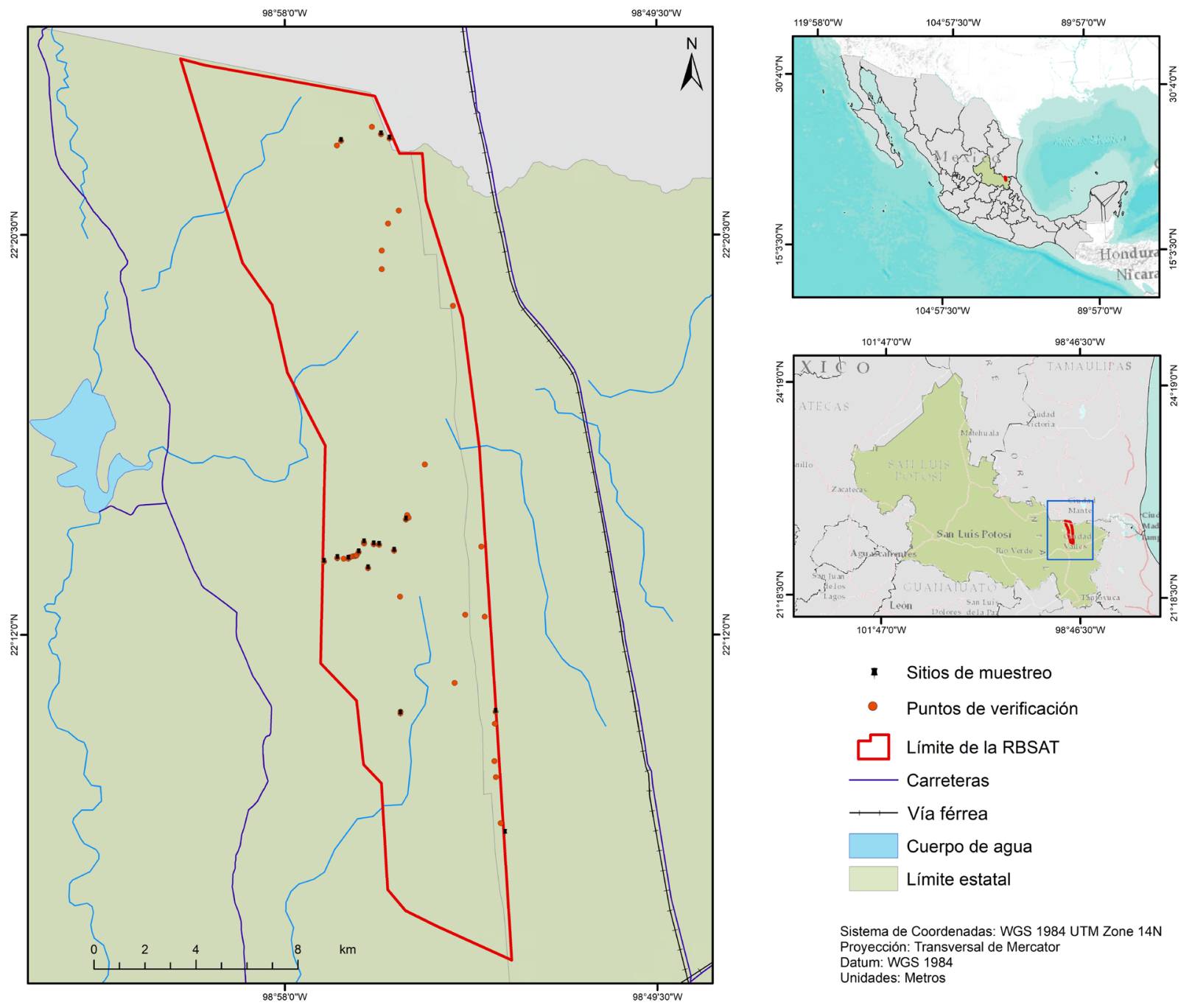

- Sitios de muestreo

- Puntos de verificación

$\square$ Limite de la RBSAT

Carreteras

$\longleftarrow$ Via férrea

Cuerpo de agua

Limite estatal

Sistema de Coordenadas: WGS 1984 UTM Zone 14N Proyección: Transversal de Mercato

Datum: WGS 1984
Unidades: Metros

Figura 1. Localización y sitios de muestreo en la RBSAT.

et al. (2018), a través de un proceso de re etiquetado de polígonos en el Sistema de Información Geográfica (SIG) ArcGis 10.3 (ESRI 2015). Posteriormente se generó una nueva capa digital con la distribución actualizada de las variantes de vegetación. A partir de la información actualizada se obtuvo la superficie por variante dentro del polígono del área núcleo de la RBSAT en hectáreas, así como la superficie para la zona de influencia.

Por otro lado, se procedió a identificar zonas susceptibles de ser incorporadas al esquema de ADVC en el futuro. Para tal efecto se consideraron como criterios para la selección: a) el régimen de tenencia de la tierra, b) la presencia y superficie de variantes de vegetación y c) la distancia al polígono del ANP, desde el centroide de cada sitio propuesto al punto más cercano del área núcleo de la RBSAT. Inicialmente, se sobrepuso la información sobre la tenencia de la tierra (RAN 2020) a la capa de información actualizada de las coberturas de vegetación y usos de suelo y se determinó la ubicación de sitios donde existieran remanentes de las variantes de vegetación fuera del área núcleo de la RBSAT. Posteriormente, se determinó la tenencia de la tierra de los sitios seleccionados, la superficie en ha de las variantes incluidas en cada uno y se calculó su distancia a la zona núcleo en el SIG. En todos los casos se consideró la posibilidad de mantener la conectividad ecológica de los sitios con el ANP de forma que se justifique su posible conservación a largo plazo. Los criterios utilizados responden a lo dispuesto en la Ley General del Equilibrio Ecológico y la Protección al Ambiente (DOF 2016) para facilitar la determinación de las características y elementos que un predio debe cumplir para ser propuesto como ADVC. 


\section{Resultados}

Composición florística. En los 17 sitios de muestreo se registraron un total de 1,636 individuos de plantas leñosas con DAP mayor a $2.5 \mathrm{~cm}$, para 92 especies. Con la revisión de los especímenes depositados en herbario colectados en la zona y los inventarios más recientes, la riqueza total de plantas leñosas de la RBSAT es de al menos 177 especies, de las cuales 154 son arbóreas o arbustivas y 23 son trepadoras leñosas (Tabla S1, Material Suplementario). Con esta riqueza de especies el IB estimado para el área es de 18.19. La familia Fabaceae fue la que presentó mayor riqueza (32 especies y 24 géneros), seguida por Euphorbiaceae (19 especies y nueve géneros), Apocynaceae (nueve especies y nueve géneros) y Malpighiaceae (ocho especies y seis géneros).
Distribución y características de las variantes de vegetación. Con base en el análisis de agrupamiento realizado con los datos del VIR de las especies se reconocen cuatro variantes de vegetación (Figura 2): selva baja caducifolia (SBC, Figura 3A), selva baja subcaducifolia (SBSC, Figura 3B), selva mediana subcaducifolia (SMSC, Figura 3C) y selva mediana subperennifolia (SMSP, Figura 3D). La distribución de las variantes de vegetación del polígono del área núcleo de la RBSAT, así como su extensión hacia la zona de influencia se muestra en la Figura 4.

Selva baja caducifolia.- Esta variante de vegetación tiene una extensión de 7,590.77 ha dentro del área núcleo e incrementa hasta $28,394.25$ ha si se incluye su continuidad hacia la zona de influencia (Tabla 1) en altitudes de 300 a $400 \mathrm{~m}$. La altura de los elementos leñosos de esta variante de vegetación va de 5 a $10 \mathrm{~m}$. Presenta una marcada domi-

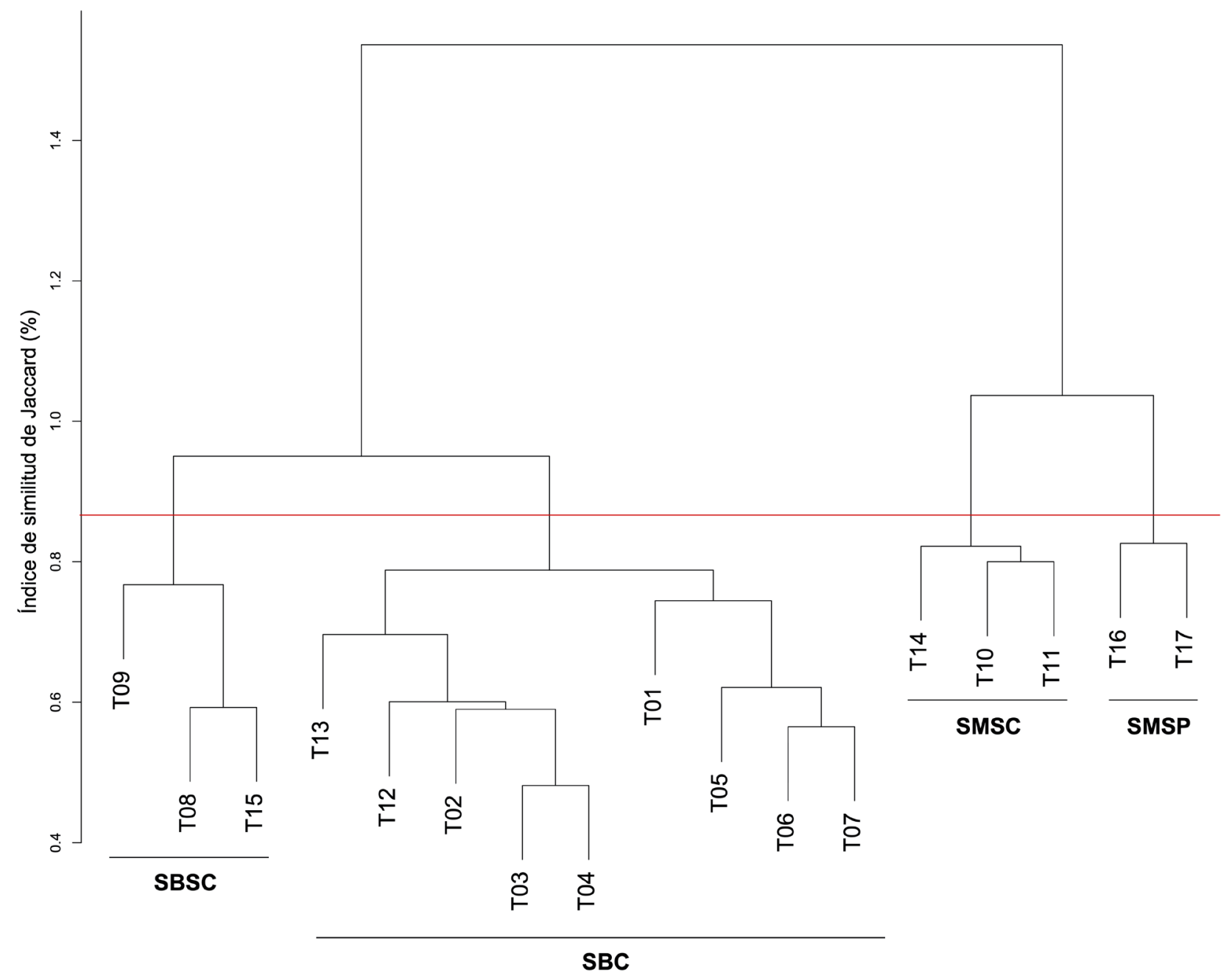

Figura 2. Dendrograma que representa las relaciones jerárquicas entre los sitios de muestreo (Índice de similitud de Jaccard). SBC: selva baja caducifolia. SBSC: selva baja subcaducifolia. SMSC: selva mediana subcaducifolia. SMSP: selva mediana subperennifolia. 
nancia de Beaucarnea inermis (S.Watson) Rose (VIR: 79.06), Croton sp. (VIR: 34.77), Euphorbia schlechtendalii Boiss (VIR: 22.80) y Drypetes lateriflora (Sw.) Krug \& Urb. (VIR: 21.90) (Tabla 2), además de otras especies tales como Pseudobombax ellipticum (Kunth) Dugand, Harpalyce arborescens A.Gray, Exostema mexicanum A.Gray, Bursera simaruba (L.) Sarg., Psidium sartorianum L. y Exostema caribaeum (Jacq.) Schult.

Selva baja subcaducifolia.- Esta variante de vegetación es la más extendida dentro de la RBSAT y cubre una superficie de 10,438.64 ha dentro del área núcleo que se incrementa hasta $13,586.89$ ha si se incluye su continuidad hacia la zona de influencia (Tabla 1), se localiza entre los 500 y $600 \mathrm{~m}$ snm en zonas con presencia de cañadas, dolinas y sumideros. La altura de los principales elementos leñosos en esta variante de vegetación es de 7 a $12 \mathrm{~m}$, aunque frecuentemente algunos individuos de Acacia californica subsp. pringlei (Rose) L. Rico alcanzan hasta 18 $\mathrm{m}$. Presenta una dominancia de Beaucarnea inermis (VIR: 102.54), Drypetes lateriflora (VIR: 51.56) y Euphorbia schlechtendalii (VIR: 21.45) (Tabla 2), además de otras especies tales como Psidium sartorianum, Pseudobombax ellipticum, Randia laetevirens Standl., Ocotea tampicensis (Meisn.) Hemsl. y Croton sp.

Selva mediana subcaducifolia.- Esta variante de vegetación registra una extensión de 2,335.08 ha dentro del área núcleo e incrementa hasta $2,377.86$ ha si se incluye su continuidad hacia la zona de influencia (Tabla 1) y se distribuye en altitudes que van de los 500 a $800 \mathrm{~m}$. Los principales elementos leñosos alcanzan una altura de 15 $\mathrm{m}$, con algunos individuos de hasta los $30 \mathrm{~m}$, como Acacia californica subsp. pringlei. Este sitio presenta dominancia de Esenbeckia runyonii C.V.Morton (VIR: 44.21), Drypetes lateriflora (VIR: 42.43), Psidium sartorianum (VIR: 35.99) y Cinnamomum tampicense (Meisn.) Kosterm. (VIR: 21.35) (Tabla 2), además de otras especies tales como Aphananthe monoica (Hemsl.) J.-F.Leroy, Randia laetevirens, Fraxinus dubia (Willd. ex Schult. \& Schult.f.) P.S.Green \& M.Nee, Wimmeria concolor Cham.
\& Schltdl., Neea psychotrioides Donn. Sm., Persea americana Mill., Ocotea tampicensis y Protium copal (Schltdl. \& Cham.) Engl.

Selva mediana subperennifolia.- Se encuentra en la parte este de la sierra y es la de menor extensión con 423.92 ha dentro del área núcleo e incrementa hasta 957.08 ha si se incluye su continuidad hacia la zona de influencia (Tabla 1), se presenta entre los 100 y $300 \mathrm{~m} \mathrm{snm}$. La altura de los principales elementos leñosos oscila entre los $10 \mathrm{a}$ $15 \mathrm{~m}$, aunque algunas especies llegan alcanzar hasta los $36 \mathrm{~m}$ como Acacia californica subsp. pringlei. Las especies con mayor dominancia fueron Brosimum alicastrum Sw. (VIR: 58.14), Acacia californica subsp. pringlei (VIR: 41.93), Aphananthe monoica (VIR: 27.78), Bursera simaruba (VIR: 26.01) y Coccoloba barbadensis Jacq. (VIR: 25.85) (Tabla 2), además de otras especies presentes como Drypetes lateriflora, Iresine interrupta Benth., Chrysophyllum mexicanum Brandegee, Ebenopsis ebano (Berland.) Barneby \& J.W.Grimes, Adelia barbinervis Cham. \& Schltdl. y Protium copal.

La comparación entre las superficies ocupadas por las variantes de vegetación entre la cartografía existente (Reyes-Hernández et al. 2018) y la capa digital generada muestra diferencias relacionadas con el incremento de la superficie de la SBC (408.32 ha) y el decremento en la superficie de la SBSC (46.16 ha), la SMSC (10.58 ha) y la $\operatorname{SMSP}(351.58 \mathrm{ha})$.

Riqueza de especies y diversidad beta. La riqueza de especies leñosas en el total del área muestreada fue más alta en la SBC (al menos 61 especies) (Tabla 3). En contraste, la SMSP obtuvo la menor riqueza en la zona de estudio con al menos 23 especies. Por otra parte, la SBC y SBSC tienen la menor tasa de reemplazo de especies (WS $=0.47$; Tabla 3), y una mayor semejanza florística (similitud de Jaccard $=0.64$; Tabla 3). En contraste con la SMSP y SBSC que tienen una mayor tasa de reemplazo (WS $=0.74$; Tabla 3 ) y presentan una menor semejanza florística (similitud Jaccard $=0.85$; Tabla 3).

Tabla 1. Superficie de las variantes de vegetación en la RBSAT y su zona de influencia.

\begin{tabular}{lcc}
\hline Variante de vegetación & Polígono de la RBSAT (ha) & $\begin{array}{c}\text { Polígono de la RBSAT y zona } \\
\text { de influencia (ha) }\end{array}$ \\
\hline Selva baja caducifolia & $7,590.77$ & $28,394.25$ \\
Selva baja subcaducifolia & $10,438.64$ & $13,586.89$ \\
Selva mediana subcaducifolia & $2,335.08$ & $2,377.86$ \\
Selva mediana subperennifolia & 423.92 & 957.08 \\
\hline
\end{tabular}



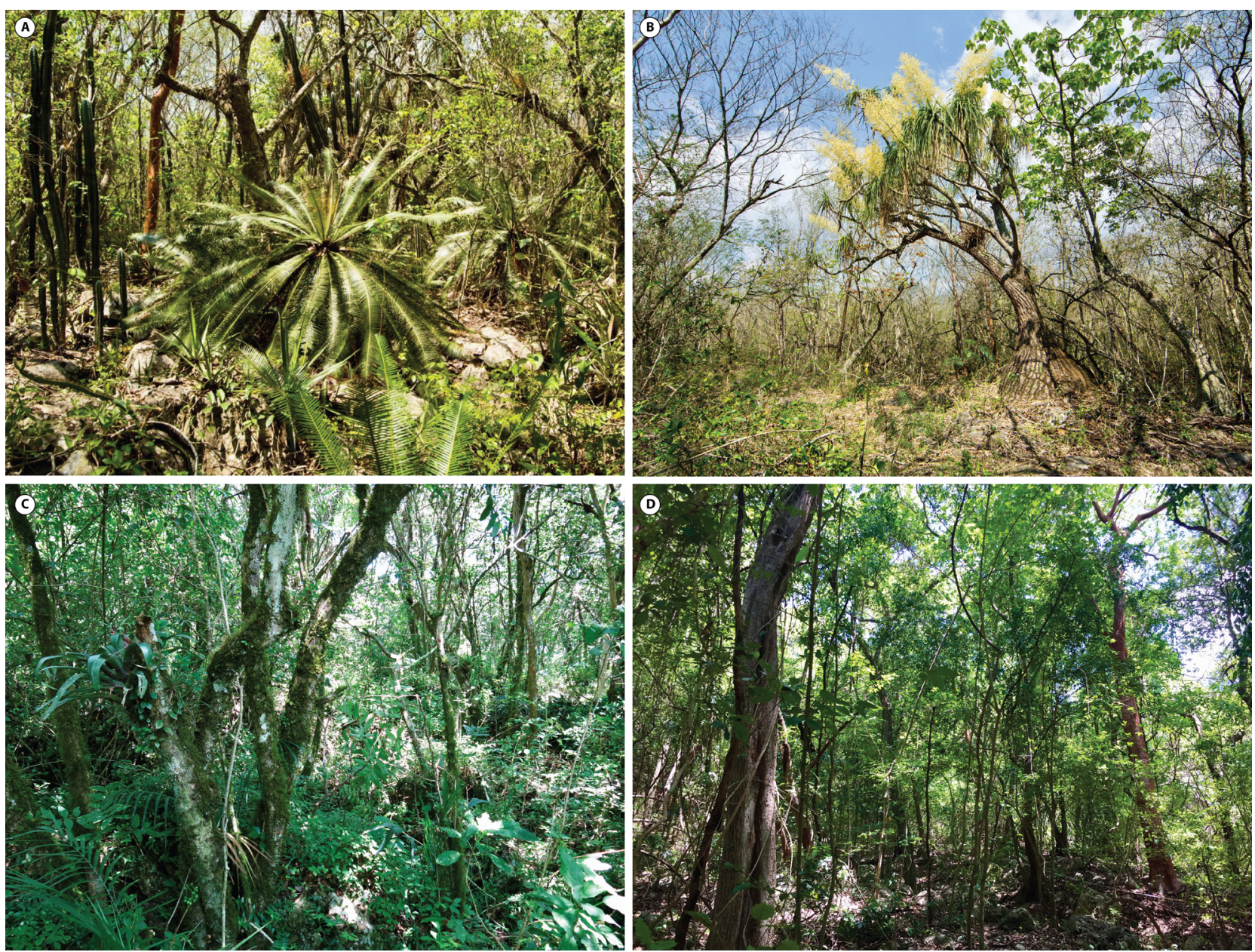

Figura 3. Variantes de vegetación de la RBSAT. A) Selva baja caducifolia; B) Selva baja subcaducifolia; C) Selva mediana subcaducifolia; D) Selva mediana subperennifolia. Fotos A, B: CONANP/RBSAT. Foto C: J.A. de Nova. Foto D: R. Gutiérrez.

Zonas con potencial para el establecimiento de ADVC. Se identificaron cinco áreas con posibilidad de ser consideradas como ADVC (Figura 5, Tabla 4). La ADVC 1 se localiza en Las Yeguas, Rancho San Diego y El Reparito, en el ejido Laguna del Mante, Ciudad Valles, a $2.5 \mathrm{~km}$ de la RBSAT, con dos tipos de tenencia de la tierra: ejidal y privada. En ella se presentan tres variantes de vegetación: SBC (1,254.42 ha), SBSC (14.31 ha) y SMSP (7.83 ha). La ADVC 2 localizada en el ejido Laguna del Mante, Ciudad Valles se encuentra a $5.13 \mathrm{~km}$ de la RBSAT; la tenencia de la tierra es ejidal y presenta tres variantes de vegetación, SBC (1,049.2 ha), SBSC (25.65 ha) y SMSP (2.88 ha). La ADVC 3 se ubica en el Rancho Dolores (Tanchipa) Tamuín a $2.56 \mathrm{~km}$ de distancia de la RBSAT, la tenencia de la tierra es ejidal de uso común y propiedad privada e incluye tres variantes de vegetación, SBC (628.92 ha), SBSC (6.66 ha) y SMSP (160.38 ha). La ADVC 4 se localiza en el ejido Las Palmas, Ciudad Valles a $2.36 \mathrm{~km}$ de distancia de la
RBSAT, la tenencia de la tierra es ejidal de uso común, y presenta cuatro variantes de vegetación, SBC (159.39 ha), SBSC (1,273.77 ha), SMSC (0.09 ha) y SMSP (6.03 ha). Finalmente, la ADVC 5 se ubica en el ejido Montecillos, Ciudad Valles a $6.28 \mathrm{~km}$ de distancia de la RBSAT. La tenencia de la tierra incluye zonas ejidales de uso común, en donde se distribuyen las tres variantes de vegetación, $\mathrm{SBC}$ (1,144.44 ha), SBSC (82.98 ha) y SMSP (1.71 ha).

\section{Discusión}

Composición y riqueza de las variantes de vegetación de la RBSAT. El conocimiento de la estructura y composición de las comunidades vegetales de ecosistemas vulnerables del Neotrópico permite generar conocimiento fundamental para el aprovechamiento y conservación de sus recursos naturales (Saxena \& Singh 1984, Shugart \& West 1980, Zamora-Crescencio et al. 2016). La relevancia estructural 
Bosques tropicales estacionalmente secos de la Sierra del Abra Tanchipa

Tabla 2. Especies leñosas con mayor valor de importancia relativa promedio (VIR) por variante de vegetación en la RBSAT. SBC: selva baja caducifolia. SBSC: selva baja subcaducifolia. SMSC: selva mediana subcaducifolia. SMSP: selva mediana subperennifolia.

\begin{tabular}{|c|c|c|c|c|}
\hline Especie & SBC & SBSC & SMSC & SMSP \\
\hline Acacia californica subsp. pringlei (Rose) L. Rico & 1.15 & 2.59 & 5.63 & 41.93 \\
\hline Aphananthe monoica (Hemsl.) J.-F.Lerov & 1.36 & - & 17.19 & 27.78 \\
\hline Beaucarnea inermis (S.Watson) Rose & 79.06 & 102.54 & - & - \\
\hline Brosimum alicastrum Sw. & - & - & - & 58.14 \\
\hline Bursera simaruba (L.) Sarg. & 8.10 & 5.71 & 1.62 & 26.01 \\
\hline Cinnamomum tampicense (Meisn.) Kosterm. & - & - & 21.35 & - \\
\hline Coccoloba barbadensis Jacq. & 2.86 & - & - & 25.85 \\
\hline Croton sp. & 34.77 & 6.49 & - & - \\
\hline Drypetes lateriflora (Sw.) Krug \&Urb. & 21.90 & 51.56 & 42.43 & 17.65 \\
\hline Esenbeckia runyonii C.V. Morton & 0.47 & 3.60 & 44.21 & - \\
\hline Euphorbia schlechtendalii Boiss. & 22.80 & 21.45 & 2.78 & - \\
\hline Psidium sartorianum (O.Berg) Nied. & 8.02 & 19.37 & 35.99 & - \\
\hline
\end{tabular}

de las especies arbóreas y arbustivas dentro de los BTES se ha resaltado en diversas investigaciones (Gillespie et al. 2000, Trejo \& Dirzo 2002, Pérez-García et al. 2012, DeNova et al. 2019), en especial el hecho de que los árboles son un grupo indicador para la selección de áreas prioritarias para la conservación (Rzedowski 1978, 1991, Wendt 1993, Ibarra-Manríquez et al. 2002, Santiago et al. 2002, Villaseñor et al. 2003, Carranza-González 2005, Cué-Bär et al. 2006). El IB de plantas leñosas de la RBSAT (18.19) coincide con lo reportado previamente por Bravo-Bolaños et al. (2016), quienes señalan que para los BTES de México la riqueza de especies de árboles y arbustos es contrastante, pero en su mayoría se reportan valores de IB entre 16.1 y 76.4 (Valiente-Banuet et al. 1995, Pérez-García et al. 2001, Godínez-Ibarra \& López-Mata 2002, Cué-Bär et al. 2006, Salas-Morales et al. 2007, López-Patiño et al. 2012, Zamora-Crescencio et al. 2016). Zamora-Crescencio et al. (2016) señalan que la variación en el número de especies por unidad de área en los BTES puede tener múltiples causas, como son el tamaño del área, el esfuerzo de muestreo, diferencias en la heterogeneidad espacial de los sitios y condiciones ambientales, entre otras. Por otro lado, se ha mencionado una relación negativa entre la riqueza de árboles y la latitud (Gentry 1982, Trejo \& Dirzo 2002), lo que puede explicar valores de diversidad de plantas leñosas más bajos en la RBSAT ya que se encuentra en el límite septentrional de los ecosistemas tropicales estacionales del Golfo de México.

La importancia de las familias Fabaceae y Euphorbiaceae en la RBSAT coincide con diversos estudios rea-

Tabla 3. Índices de diversidad beta entre las variantes de vegetación de la RBSAT. Diagonal superior: Diversidad beta (Índice de Wilson y Shmida). Diagonal inferior: Índice de similitud de Jaccard. Riqueza de especies por variante de vegetación (números en negritas). SBC: selva baja caducifolia. SBSC: selva baja subcaducifolia. SMSC: selva mediana subcaducifolia. SMSP: selva mediana subperennifolia.

\begin{tabular}{lcccc}
\hline & SMSC & SBSC & SBC & SMSP \\
\hline SMSC & $\mathbf{3 4}$ & 0.59 & 0.68 & 0.72 \\
SBSC & 0.75 & $\mathbf{3 0}$ & 0.47 & 0.74 \\
SBC & 0.81 & 0.64 & $\mathbf{6 1}$ & 0.69 \\
SMSP & 0.84 & 0.85 & 0.82 & $\mathbf{2 3}$ \\
\hline
\end{tabular}


lizados en los BTES de México (Trejo \& Dirzo 2002, Pineda-García et al. 2007, Williams-Linera \& Lorea 2009, Rzedowski \& Calderón de Rzedowski 2013, Méndez-Toribio et al. 2014, Hernández-Ramírez \& García Méndez 2015, Bravo-Bolaños et al. 2016, De-Nova et al. 2019) y confirma su predominio en el Neotrópico ya señalado por Gentry (1995), Gillespie (2000) y Pennington et al. (2006). Otras familias importantes por su riqueza de especies leñosas en los BTES de México son Moraceae y Sapotaceae (Trejo 2010, Rzedowski \& Calderón de Rzedowski $\underline{2013}$, Villaseñor 2016, Bravo-Bolaños et al. 2016), igualmente importantes para la RBSAT. El $78.9 \%$ de las fa- milias de especies leñosas de la RBSAT incluye entre una y dos especies, lo cual coincide con la diversidad florística reportada para otros estudios de BTES del Neotrópico donde mencionan un alto número de familias y géneros representados por pocas especies (Palacios-Wassenaar et al. 2018). Por ejemplo, Trejo \& Dirzo (2002) señalan que la diversidad florística de estos bosques también se refleja a nivel de géneros con una relación de 1.17 especies por género registradas para los BTES de México.

Diversidad de las variantes de vegetación en la RBSAT. Los valores de diversidad beta registrados entre las varian-

\section{Uso de suelo y Vegetación \\ Agricultura \\ Pastizal inducido \\ Selva baja caducifolia \\ Selva baja subcaducifolia \\ Selva mediana subcaducifolia \\ Selva mediana subperennifolia \\ Vegetación secundaria \\ Cuerpo de agua \\ Límite de la RBSAT \\ Área de influencia al ANP \\ Carreteras \\ Localidades urbanas \\ Límite estatal}

Sistema de Coordenadas: WGS 1984 UTM Zone $14 \mathrm{~N}$

Proyección: Transversal de Mercator

Datum: WGS 198

Unidades: Metros

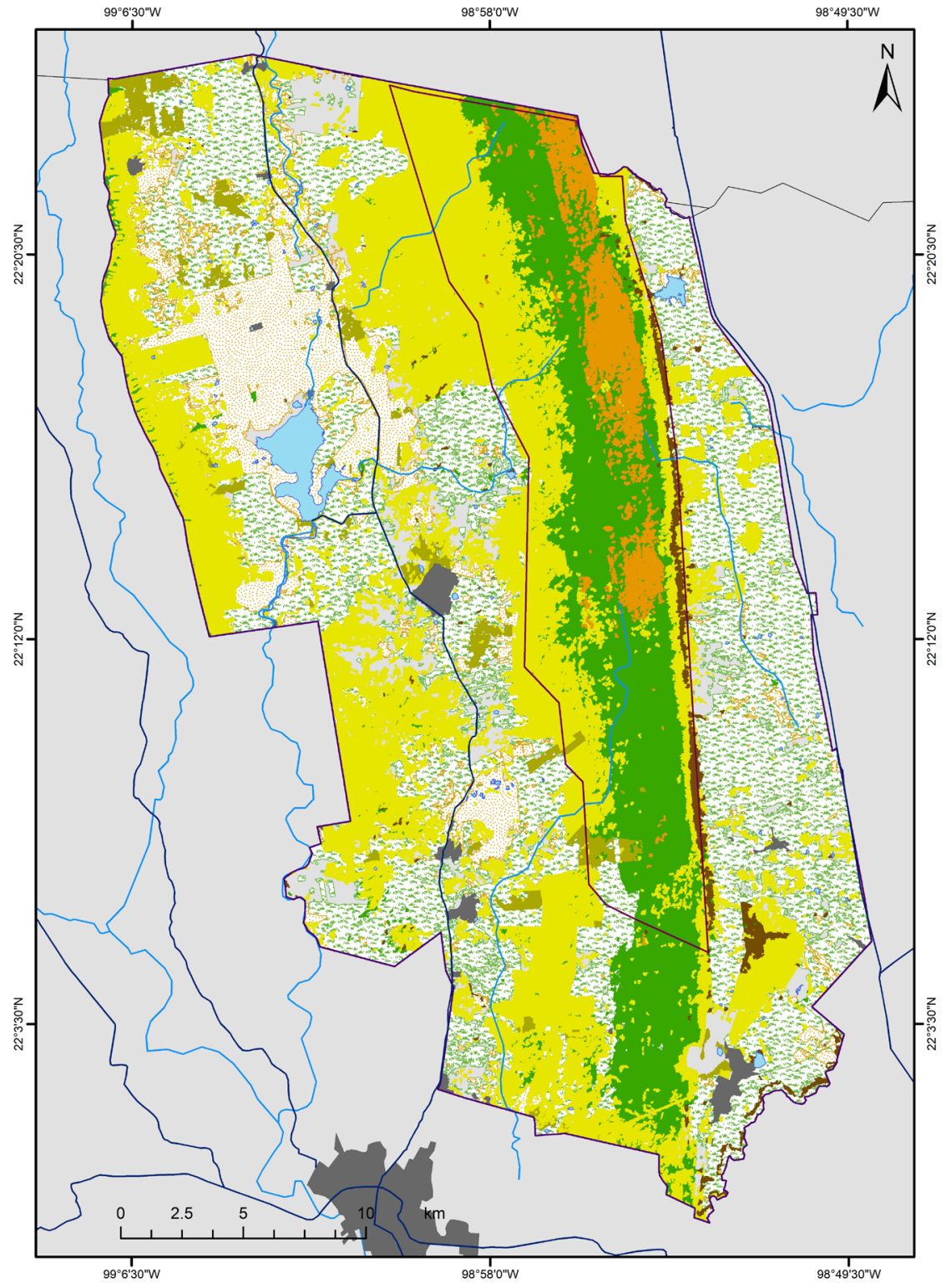

Figura 4. Distribución de las variantes de vegetación en la RBSAT y su zona de influencia. 
Bosques tropicales estacionalmente secos de la Sierra del Abra Tanchipa

Tabla 4. Sitios propuestos como ADVC en la zona de influencia de la RBSAT. SBC: selva baja caducifolia. SBSC: selva baja subcaducifolia. SMSC: selva mediana subcaducifolia. SMSP: selva mediana subperennifolia.

\begin{tabular}{|c|c|c|c|c|c|c|}
\hline ADVC & Municipio & Ejidos o propiedades & $\begin{array}{l}\text { Tenencia de } \\
\text { la tierra }\end{array}$ & $\begin{array}{l}\text { Superficie } \\
\text { Total (ha) }\end{array}$ & $\begin{array}{l}\text { Variante de vegetación } \\
\text { (superficie en ha) }\end{array}$ & $\begin{array}{l}\text { Distancia a la zona } \\
\text { núcleo }(\mathbf{k m})\end{array}$ \\
\hline 1 & Ciudad Valles & $\begin{array}{l}\text { Laguna del Mante, } \\
\text { Las Yeguas, Ran- } \\
\text { cho San Diego, El } \\
\text { Reparito }\end{array}$ & $\begin{array}{l}\text { Ejidal y } \\
\text { privado }\end{array}$ & $1,276.56$ & $\begin{array}{c}\operatorname{SBC}(1,254.42) \\
\operatorname{SBSC}(14.31) \\
\operatorname{SMSP}(7.83)\end{array}$ & 2.50 \\
\hline 2 & Ciudad Valles & Laguna del Mante & Ejidal & $1,078.20$ & $\begin{array}{c}\text { SBC }(1,049.67) \\
\operatorname{SBSC}(25.65) \\
\operatorname{SMSP}(2.88)\end{array}$ & 5.13 \\
\hline 3 & Tamuín & $\begin{array}{l}\text { Rancho Dolores } \\
\text { (Tanchipa) }\end{array}$ & $\begin{array}{l}\text { Comunal y } \\
\text { privado }\end{array}$ & 795.96 & $\begin{array}{l}\text { SBC (628.92) } \\
\text { SBSC (6.66) } \\
\operatorname{SMSP}(2.88)\end{array}$ & 2.56 \\
\hline 4 & Ciudad Valles & Las Palmas & $\begin{array}{l}\text { Comunal y } \\
\text { ejidal }\end{array}$ & $1,439.28$ & $\begin{array}{c}\operatorname{SBC}(159.39) \\
\operatorname{SBSC}(1,273.77) \\
\operatorname{SMSC}(0.09) \\
\operatorname{SMSP}(6.03)\end{array}$ & 2.36 \\
\hline 5 & Ciudad Valles & Montecillos & $\begin{array}{c}\text { Comunal y } \\
\text { ejidal }\end{array}$ & $1,229.13$ & $\begin{array}{c}\text { SBC }(1,144.44) \\
\operatorname{SBSC}(82.98) \\
\operatorname{SMSP}(1.71)\end{array}$ & 6.28 \\
\hline
\end{tabular}

tes de vegetación para la RBSAT están en un rango de 0.47 a 0.74 , los cuáles se encuentran dentro de lo reportado para otros sitios con BTES en México. Por ejemplo, Balvanera et al. (2002) reporta valores entre 0.30 y 0.79 para Chamela, Jalisco, mientras que Álvarez-Yépiz et al. (2008) y Williams-Linera \& Lorea (2009) reportan valores entre 0.49 y 0.68 y 0.13 y 0.62 respectivamente. Para el noroeste de México, y Bravo-Bolaños et al. (2016) reportan valores entre 0.63 y 0.96 para Bahía de Banderas, Nayarit. El hecho de que la diversidad florística de los BTES mexicanos es alta, tanto a nivel local como regional, tiene una implicación importante desde el punto de vista de la conservación, como lo señalan Trejo \& Dirzo (2002), quienes además destacan la necesidad de implementar una red de numerosas reservas distribuidas en todo el país para la protección de este tipo de bosque en México.

Respecto a las variantes de vegetación, las especies con el valor más alto de VIR para la SBC fueron Beaucarnea inermis, Croton sp., Euphorbia schlechtendalii y Drypetes lateriflora y para la SBSC B. inermis, D. lateriflora y $E$. schlechtendalii, lo que coincide con estudios anteriores donde señalan que la presencia y dominancia de especies como $B$. inermis es característica de la SBC, y que indica la transición entre la SMSC y la SBC (Miranda \& Hernández-X 1963, Castillo-Campos 1995, Pineda-García et al. 2007, Palacios-Wassenaar et al. 2018). Por otra parte, Rubio-Méndez et al. (2018) resaltan la importancia de Beaucarnea inermis dentro de la RBSAT de acuerdo con sus valores de VIR, esto debido principalmente a su gran dominancia en la SBC y SBSC asociada a su área basal. Para el caso de la SMSC, con condiciones ligeramente más húmedas, resalta la presencia y dominancia de Esenbeckia runyonii, Psidium sartorianum y Cinnamomum tampicense. Por su parte, D. lateriflora es frecuente tanto en la SBC y SBSC. La SMSP, que es la variante de vegetación con mayor humedad, está dominada por especies como Brosimum alicastrum, Acacia californica subsp. pringlei, Aphananthe monoica, Bursera simaruba y Coccoloba barbadensis.

Las variantes de vegetación determinadas en el presente estudio coinciden con las definidas, con un método distinto por Reyes-Hernández et al. (2018). Sin embargo, el trabajo de campo y el análisis de las variantes de vegetación realizado permitió la actualización de la capa digital de cobertura de vegetación y su posterior contraste con la cartografía generada previamente por dichos au- 
tores. Lo anterior, hizo posible la representación espacialmente explicita de la ubicación actualizada de las variantes SBC, SBSC, SMSC y SMSP en el ANP y su zona de influencia. Al contrastar la información, se destaca el incremento de 408.32 ha para la SBC y el decremento de 351 ha para la SMSP, situación que demandará de un análisis específico para entender los procesos subyacentes. Sin duda, la actualización de la ubicación de los polígonos donde se distribuyen las variantes de BTES favorecerá su manejo en la zona.

Áreas con potencial para la conservación. La región de la Huasteca potosina donde se encuentra la RBSAT, ha perdido alrededor de 2,520 ha de selvas bajas en los últimos 26 años, por los procesos de cambio de uso del suelo, la deforestación, y la degradación por actividades agro- pecuarias no sustentables (Ramírez-Albores 2007, Vergara-Paternina et al. 2017, Reyes-Hernández et al. 2018, Sahagún-Sánchez \& Reyes-Hernández 2018). Estudios recientes indican que, si bien dentro del polígono de protección de la RBSAT solo se deforestaron 37 ha de BTES entre 1996 y 2016 que representa una tasa de deforestación anual bastante baja (menor al 0.01\%) (Vázquez-Villa et al. 2020), la dinámica de transformación que ocurre en el área de influencia es bastante mayor (Reyes-Hernández et al. 2018). Los BTES presentan una importante riqueza de especies, de las cuales un elevado número son endémicas, y exhibe un importante recambio que incrementa su diversidad (Luna et al. 2004, Berlanga-Robles et al. 2018). Por lo anterior, el conocimiento de la composición y estructura de la vegetación resulta prioritario ante la amenaza por las alarmantes tasas de pérdida de estos ecosistemas (ㅁortillo-

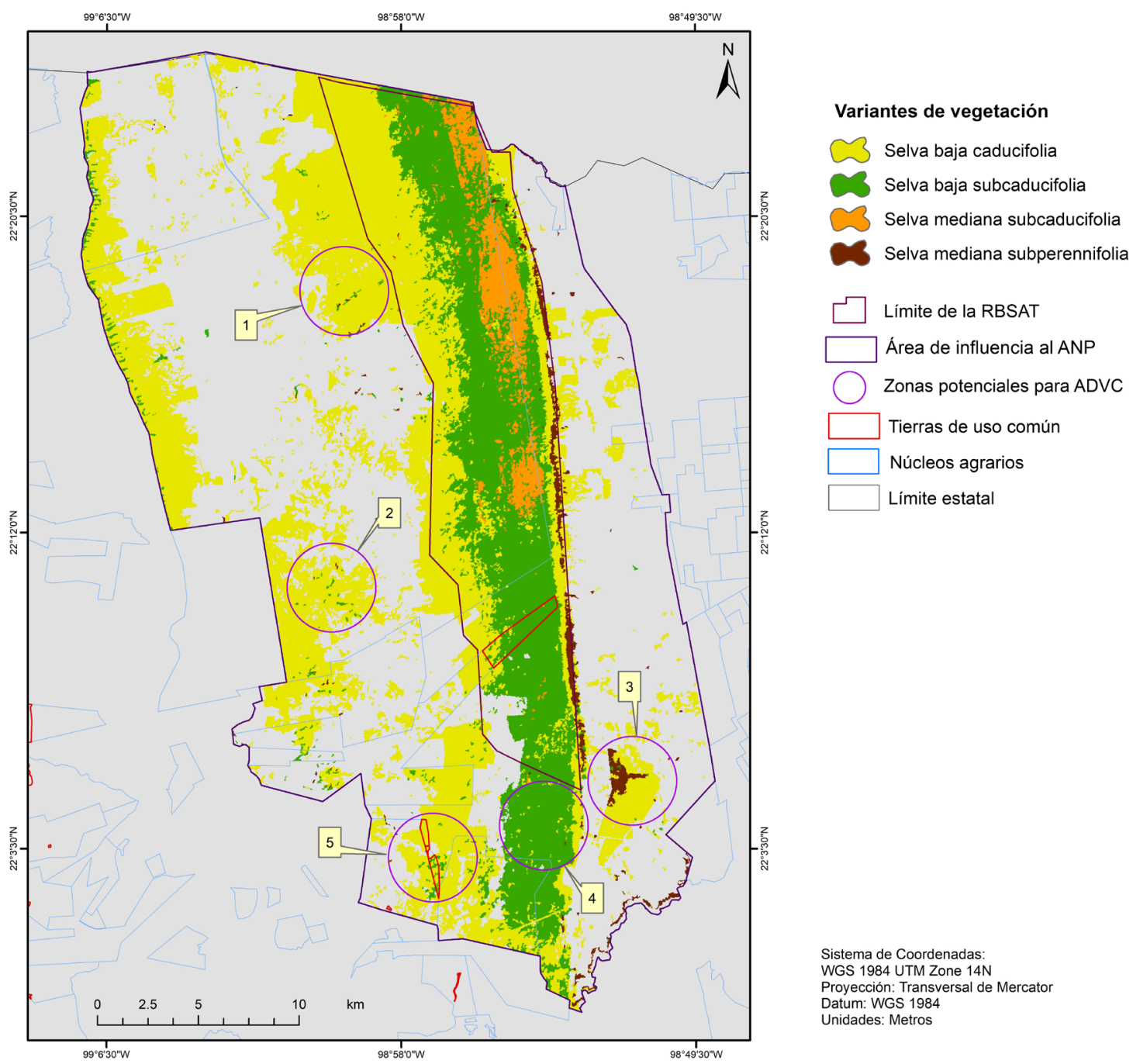

Figura 5. Sitios propuestos como Áreas Destinadas Voluntariamente a la Conservación en la zona de influencia de la RBSAT. 
Quintero \& Sánchez-Azofeifa 2010). La información actualizada de la distribución de las diferentes variantes de vegetación es fundamental para planificar y administrar adecuadamente este espacio protegido por parte de los tomadores de decisiones.

Las ADVC promueven la participación directa de las comunidades en la gestión ambiental y reconocen la legitimidad de los esfuerzos de conservación por parte de los propietarios, sean de ejidos, comunidades agrarias o de personas físicas o morales (DOF 2016). A nivel nacional, la extensión cubierta por ADVC es de 511,338.87 ha en 22 estados del país (Bertzky et al. 2012, CONANP 2018b, SEMARNAT-CONANP 2016, Silva-Aparicio et al. 2018). Sin embargo, no existe ninguna ADVC en el estado de San Luis Potosí (CONANP 2020), por lo que plantear su establecimiento constituye una oportunidad para expandir los esfuerzos de conservación en el ámbito de la RBSAT. La delimitación formal de alguna ADVC podría favorecer el mantenimiento de la matriz de vegetación y con ello la permanencia de las cubiertas vegetales de interés y la biodiversidad que soportan. Además, estudios recientes indican la necesidad de ampliar las acciones de conservación a las áreas con remanentes de vegetación debido a que constituyen corredores por donde se desplazan especies emblemáticas e indicadoras como el jaguar (Panthera onca), que se distribuyen en las inmediaciones de la RBSAT (Martínez-Hernández et al. 2017).

Es necesario desarrollar estrategias de manejo que permitan asegurar la permanencia y conservación a largo plazo en el ANP (Ceballos et al. 2010); en particular, debido a que continúan los procesos de fragmentación y degradación de los remanentes de BTES en la zona (Reyes-Hernández et al. 2018). Los trabajos orientados a la conservación de los recursos naturales en cogestión con los actores locales (ejidatarios y propietarios), son un factor clave para lograr los objetivos de conservación, y uno de los ejes de acción prioritaria para la CONANP (CONANP 2020). Impulsar el establecimiento de las $\mathrm{ADVC}$ aquí identificadas puede resultar una alternativa viable. Asimismo, la aplicación de nuevos modelos de gestión para las áreas naturales en la región, basados en esquemas de gobernanza ambiental y la colaboración con organizaciones internacionales que provean apoyo financiero, pueden significar una oportunidad para la conservación de los BTES en la región (Vázquez-Villa et al. 2020). Los BTES en el ámbito de la RBSAT constituyen un patrimonio de la biodiversidad regional, por lo que conocer su estructura y distribución actual permitirá reestructurar los planes de manejo a corto y mediano plazo, de forma que se garantice su permanencia y la consecuente provisión de servicios ecosistémicos.

\section{Agradecimientos}

Agradecemos al Consejo Nacional de Ciencia y Tecnología, a la Comisión Nacional para el Conocimiento y Uso de la Biodiversidad, la Secretaría de Educación Pública, la Comisión Nacional de Áreas Naturales Protegidas y SEMARNAT por el apoyo económico e infraestructura que permitió realizar esta investigación, particularmente a los proyectos CONACYT CB-2014/243454, CONABIO FB1829/ PJ029/17 y SEP-PRODEP $103.5 / 13 / 6575$ otorgados a JADN. La primera autora agradece el apoyo de CONACYT (Beca de maestría 931690) y al Posgrado en Ciencias Agropecuarias de la FAyV de la UASLP. Al Ing. Alejandro Durán Fernández, director de la RBSAT, por todas las facilidades y el apoyo durante las investigaciones realizadas en el ANP. Agradecemos el apoyo en campo de Don Hermelindo Guzmán Antonia, Luis Enrique Martínez, Mercedes Elizabeth Ramírez Elías, Maywalida Montenegro Herrera. A Don José García-Pérez por la identificación y preparación de especímenes botánicos. A Erika Galarza y Erick Omar Martínez por su apoyo en la elaboración de los mapas.

\section{Material suplementario}

El material suplemntario de este artículo puede contrarse en: https://doi.org/10.17129/botsci.2771

\section{Literatura citada}

Aguilar-Rivera N, Galindo-Mendoza G, Fortanelli-Martínez J, Contreras-Servin C. 2010. Evaluación multicriterio y aptitud agroclimática del cultivo de caña de azúcar en la región de Huasteca. México. Corpoica 11: 144-155. DOI: https://doi.org/10.21930/rcta.vol11 num2_art:207

Alanís-Rodríguez E, Aranda-Ramos R, Mata-Balderas JM, Canizales-Velázquez PA, Jiménez-Pérez J, UvalleSauceda JI, Valdecantos-Dema A, Ruiz-Bautista MG. 2010. Riqueza y diversidad de especies leñosas del bosque tropical caducifolio en San Luis Potosí, México. Ciencia UANL 13: 287-294.

Álvarez-Yépiz JC, Martínez-Yrízar A, Búrquez A, Lindquist C. 2008. Variation in vegetation structure and soil properties related to land use history of old-growth and secondary tropical dry forests in northwestern Mexico. Forest Ecology and Management 256: 355-366. DOI: https://doi.org/10.1016/j.foreco.2008.04.049 
Anderson MJ, Crist TO, Chase JM, Vellend M, Inouye BD, Freestone AL, Sanders NJ, Cornell HV, Comita LS, Davies KF, Harrison SP, Kraft NJB, Stegen JC, Swenson NG. 2011. Navigating the multiple meanings of beta diversity: a roadmap for the practicing ecologist. Ecology Letters 14: 19-28. DOI: https://doi. org/10.1111/j.1461-0248.2010.01552.x

Arriaga-Cabrera L, Aguilar V, Espinoza J. 2009. Regiones Prioritarias y planeación para la Conservación de la Biodiversidad. In: Comisión Nacional para el Conocimiento y Uso de la Biodiversidad, ed. Capital Natural de México, Vol. II, Estado de conservación y tendencias de cambio. México, DF: Comisión Nacional para el Conocimiento y Uso de la Biodiversidad, pp. 433-457. ISBN:978-607-7607-08-3

Balvanera P, Lott E, Segura G, Siebe, C, Islas A. 2002. Patterns of $\beta$-diversity in a Mexican tropical dry forest. Journal of Vegetation Science 13: 145-158. DOI: https://doi.org/10.1111/j.1654-1103.2002.tb02034.x

Becerra JX. 2005. Timing the origin and expansion of the Mexican tropical dry forest. Proceedings of the National Academy of Sciences of the United States of America 102: 10919-10923. DOI: https://doi.org/10.1073/ pnas.0409127102

Berlanga-Robles C, Cervantes-Escobar A, MurúaFigueroa E. 2018. Estacionalidad y tendencias del bosque tropical caducifolio de la cuenca Piaxtla-ElotaQuelite y el área protegida Meseta de Cacaxtla, México. Madera y Bosques 24: e2431576. DOI: https://doi. org $/ 10.21829 / \mathrm{myb} .2018 .2431576$

Bertzky B, Corrigan C, Kemsey J, Kenney S, Ravilious C, Besançon C, Burgess N. 2012. Protected Planet Report 2012: Seguimiento del progreso de las metas globales de las áreas protegidas. Gland, Switzerland: IUCN; Cambridge, UK: UNEP-WCMC. ISBN: 978-92-807-3294-8.

Brooks TM, Bakarr MI, Boucher T, Da Fonseca GA, Hilton-Taylor C, Hoekstra JM, Moritz T, Olivieri S, Parrish J, Pressey R L. 2004. Coverage provided by the global protected-area system: Is it enough?. Bioscience 54: 1081-1091. DOI: https://doi.org/10.1641/00063568(2004)054[1081:CPBTGP]2.0.CO;2

Bravo-Bolaños O, Sánchez-González A, De NovaVázquez J, Pavón-Hernández N. 2016. Composición y estructura arbórea y arbustiva de la vegetación de la zona costera de Bahía de Banderas, Nayarit, México. Botanical Sciences 94: 1-21. DOI: https://doi. org $/ 10.17129 /$ botsci.461

Calderón J, Moreno C. 2019. Diversidad beta como disimilitud: su partición en componentes de recambio y diferencias en riqueza. In: Moreno CE, ed. La Biodiversidad en un Mundo Cambiante: Fundamentos Teóricos y Metodológicos para su Estudio. Ciudad de México: Universidad Autónoma del Estado de Hidalgo/Libermex, pp. 203-222. ISBN: 9786074825985
Carranza-González E. 2005. Biodiversidad. Angiospermas. In: Villaseñor-Villaseñor L. ed. La Biodiversidad en Michoacán. Estudio de Estado. Morelia, México: Comisión Nacional para el Conocimiento y Uso de la Biodiversidad, Gobierno del Estado de Michoacán, Secretaría de Urbanismo y Medio Ambiente, Universidad Michoacana de San Nicolás de Hidalgo, pp. 73-75. ISBN: 970-900-028-4

Castillo-Campos G. 1995. Ecología del paisaje del municipio de Jalcomulco, Veracruz. MSc. Thesis. Universidad Nacional Autónoma de México.

Ceballos G, García A. 1995. Conserving Neotropical biodiversity: the role of dry forest in western Mexico. Conservation Biology 9: 1349-1356.

Ceballos G, Martínez L, García Á, Bezaury Creel J, Dirzo R. 2010. Diversidad, amenazas y áreas prioritarias para la conservación de las selvas secas del Pacífico de México. México, DF: Comisión Nacional para el Conocimiento y Uso de la Biodiversidad. ISBN: 970-9000-38-1

Castillo-Gómez HA. 2015. Flora vascular, vegetación y plantas útiles del Cañón del Espinazo del Diablo. San Luis Potosí, México. MSc. Thesis. Universidad Autónoma de San Luis Potosí.

Challenger A, Soberón J. 2008. Los ecosistemas terrestres. In: Sarukhán J, ed. Capital Natural de México, vol. I: Conocimiento Actual de la Biodiversidad. México, DF: Comisión Nacional para el Conocimiento y Uso de la Biodiversidad. México. ISBN: 978-607-7607-03-8

CONANP [Comisión Nacional de Áreas Naturales Protegidas] 2014. Programa de manejo. Reserva de la Biosfera Sierra del Abra Tanchipa. México: Secretaría de Medio Ambiente y Recursos Naturales/Comisión Nacional de Áreas Naturales Protegidas. https://simec. conanp.gob.mx/pdf libro pm/147 libro pm.pdf (accessed November 15, 2019).

CONANP. 2018a. 100 años de conservación en México: Áreas Naturales. Ciudad de México: Comisiaón Nacional para el Conocimiento y Uso de la Biodiversidad. https:// www.conanp.gob.mx/pdf/100AñosConservación.pdf (accessed January 15, 2020).

CONANP. 2018b. Memoria documental: entrega a recepción y rendición de cuentas 2012-2018, ADVC (Otras modalidades de conservación). Ciudad de México: Comisión Nacional para el Conocimiento y Uso de la Biodiversidad. https://www.conanp.gob.mx/InformeRendicion/Memoriadocumental14.pdf (accessed January 15, 2020).

CONANP. 2020. Listado de Áreas Destinadas Voluntariamente a la conservación. https://advc.conanp.gob. $\mathrm{mx} / \mathrm{https}$-advc-conanp-gob-mx-wp-content-uploads2021-03-listado363-advc-marzo-2021-pdf/ (accessed January 15, 2020).

Cué-Bär EM, Villaseñor JL, Arredondo-Amezcua L, Cornejo-Tenorio G, Ibarra-Manrríequez G. 2006. La 
flora arbórea de Michoacán, México. Boletín de la Sociedad Botánica de México 78: 47-81. DOI: https://doi. org/10.17129/botsci.1721

De-Nova JA, Montero JC, Medina-Lemos R, Rosell J, Weeks A, Olson ME, Eguiarte LE, Magallon S. 2012. Insights into the historical construction of species-rich Mesoamerican Seasonally Dry Tropical Forests: the diversification of Bursera (Burseraceae, Sapindales). New Phytologist 193: 276-287. DOI: https://doi. org/10.1111/j.1469-8137.2011.03909.x

De-Nova JA, Castillo-Lara P, Salinas-Rodríguez MM, Fortanelli-Martínez J, Mora-Olivo A. 2018. Los bosques tropicales estacionales. In: Reyes-Hernández H, De-Nova JA y Durán FA, eds. Reserva de la Biosfera Sierra del Abra Tanchipa. Biodiversidad y Acciones para su Conservación. San Luis Potosí, México: Universidad Autónoma de San Luis Potosí-Comisión Nacional de Áreas Natutales Protegidas-Fondo Mexicano para la Conservación de la Naturaleza-Universidad Autónoma de Tamaulipas. pp. 59-78. ISBN: 978607-535-054-7

De-Nova JA, González-Trujillo R, Castillo-Lara P, Fortanelli-Martínez J, Mora-Olivo A, Salinas-Rodríguez M. 2019. Inventario florístico de la Reserva de la Biosfera Sierra del Abra Tanchipa, San Luis Potosí, México. Botanical Sciences 97: 761-788. DOI: https://doi. org/10.17129/botsci.2285

Dick CW, Wright J. 2005. Tropical mountain cradles of dry forest diversity. Proceedings of the National Academy of Sciences, USA 102: 10757-10758. DOI: https:// doi.org/10.1073/pnas.0505013102

DOF [Diario Oficial de la Federación] 1994. Decreto por el que se declara como área natural protegida, con el carácter de reserva de la biosfera, la región conocida como Sierra del Abra Tanchipa, ubicada en los municipios de Ciudad Valles y Tamuín, Estado de San Luis Potosí. México, DF. https://www.dof.gob.mx/nota detalle popup.php?codigo $=5320249$

DOF. 2016. Ley General del Equilibrio Ecológico y la Protección al Ambiente, Título Segundo, Capítulo I, Sección II, Artículo 46, Fracción XI, y del 47 al 55 BIS. México, DF. https://www.gob.mx/profepa/documentos/ley-general-del-equilibrio-ecologico-y-la-proteccion-al-ambiente-63043 (Accessed 7 May, 2020).

Durán-Fernández A. 2018. Antecedentes de la Reserva de la Biosfera Sierra del Abra Tanchipa In: Reyes-Hernández H, De-Nova JA, Durán-Fernández A, eds. Reserva de la Biosfera Sierra del Abra Tanchipa. Biodiversidad y Acciones para su Conservación. San Luis Potosí, México: Universidad Autónoma de San Luis PotosíComisión Nacional de Áreas Naturales ProtegidasFondo Mexicano para la Conservación de la Naturaleza-Universidad Autónoma de Tamaulipas. pp. 11-42. ISBN: 978-607-535-054-7
Espinosa CI, de la Cruz M, Luzuriaga AL, Escudero A. 2012. Bosques tropicales secos de la región Pacífico Ecuatorial: diversidad, estructura, funcionamiento e implicaciones para la conservación. Ecosistemas 21: 167-179. DOI: https://doi.org/10.7818/ECOS.35

ESRI [Environmental Systems Research Institute]. 2015. ArcGIS 10.3. https://www.esri.com/ (Accessed December 19, 2019).

García E. 2004. Modificaciones al Sistema de Clasificación Climática de Köppen para adaptarlo a las condiciones de la República Mexicana. México, DF: Instituto de Geografía, Universidad Nacional Autónoma de México. ISBN: 9703210104

Gentry AH. 1982. Patterns of Neotropical plant species diversity. Evolutionary Biology 15: 1-84. DOI: https:// doi.org/10.1007/978-1-4615-6968-8

Gentry AH. 1988. Changes in plant community diversity and floristic composition on environmental and geographical gradients. Annals of the Missouri Botanical Garden 75: 1-34. DOI: https://doi.org/10.2307/2399464

Gentry AH. 1995. Diversity and floristic composition of neotropical dry forests. In: Bullock SH, Mooney HA, Medina E, eds. Seasonally Dry Tropical Forests. Cambridge, Cambridge University Press, pp. 146-194. ISBN: ISBN 0521435145

Gillespie TW, Grijalva A, Farris CN. 2000. Diversity, composition, and structure of tropical dry forests in Central America. Plant Ecology 147: 37-47. DOI: https://doi.org/10.1023/A:1009848525399

Godínez-Ibarra O, López-Mata L. 2002. Estructura, composición, riqueza y diversidad de árboles tropicales en tres muestras de selva mediana subperennifolia. Anales del Instituto de Biología UNAM, Serie Botánica 73: 283-314.

Hammer Ø, Harper DAT, Ryan PD. 2001. PAST: Paleontological statistics software package for education and data analysis. Paleontologia Electronica 4: 1-9.

Hernández-Ramírez AM, García-Méndez S. 2015. Diversidad, estructura y regeneración de la selva tropical estacionalmente seca de la Península de Yucatán, México. Revista de Biología Tropical 63: 603-616.

Ibarra-Manríquez G, Villaseñor J, Durán R, Meave J. 2002. Biogeographical analysis of the tree flora of the Yucatan Peninsula. Journal of Biogeography 29: 17-29. DOI: https://doi.org/10.1046/j.13652699.2002.00648.x

INEGI [Instituto Nacional de Estadística y Geografía]. 2009. Guía para la interpretación de cartografía: uso del suelo y vegetación. Escala 1:250 000 Serie III. Aguascalientes, México: Instituto Nacional de Estadística, Geografía e Informática. ISBN 978-607494-015-2.

INEGI. 2002. Síntesis de Información Geográfica del estado de San Luis Potosí, cartografía escala 1: 700,000. 
Aguascalientes, México Instituto Nacional de Estadística, Geografía e Informática.

Leija-Loredo EG, Valenzuela-Ceballos S, Valencia-Castro M, Jiménez-González G, Castañeda-Gaytán G, ReyesHernández H, Mendoza ME. 2020. Análisis de cambio en la cobertura vegetal y uso del suelo en la región centro-norte de México. El caso de la cuenca baja del río Nazas. Ecosistemas 29: 1826. DOI: https://doi. org/10.7818/ECOS.1826

Lillesand TM, Kiefer RW, Chipman JW. 2004. Remote sensing and image interpretation. New Jersey, USA: John Wiley. Hoboken. ISBN: 978-0471152279

López-Patiño EJ, López-Sandoval JA, Beltrán-Retis AS, Aguilera-Gómez LI. 2012. Composición de la flora arbórea en el Área Natural Protegida Tenancingo-Malinalco-Zumpahuacán, Estado de México, México. Polibotánica 34: 51-98.

Lott EJ, Atkinson TH. 2002. Biodiversidad y fitogeografía de Chamela-Cuitxmala, Jalisco. In: Nogera FA, VegaRivera JH, García-Aldrete AN, Quesada-Avendaño M, eds. Historia Natural de Chamela. México, DF: Universidad Nacional Autónoma de México. ISBN: 9703205208, 9789703205202

Luna I, Morrone JJ, Espinosa D. 2004. Biodiversidad de la Sierra Madre Oriental. México, DF: Las Prensas de Ciencias. ISBN: 9703215262,9789703215263

Maass JM, Burgos A. 2011. Water Dynamics at the Ecosystem Level in Seasonally Dry Tropical Forests. In: Dirzo R, Young H, Mooney HA, Ceballos G, eds. Seasonally Dry Tropical Forests: Ecology and Conservation. Washington, DC, USA: Island Press. pp. 141-156. DOI: https://doi.org/10.5822/978-1-61091021-7 9

Martínez-Hernández A, Rosas-Rosas OC, TarangoArámbula LA, Benítez-Alemán HE. 2017. Abundance of some mesopredator preys in the Sierra del Abra Tanchipa Biosphere Reserve and adjacent areas, San Luis Potosí, Mexico. Revista Chapingo Serie Zonas Áridas 16: 37-49. DOI: https://doi.org/10.5154/r.rchsza.2017.10.005

Mas JF. 2005. Change estimates by map comparison: A method to reduce erroneous changes due to positional error. Transactions in GIS 9: 619-629. DOI: https://doi. org/10.1111/j.1467-9671.2005.00238.x

Meave JA, Romero-Romero MA, Salas-Morales SH, Pérez-García, EA, Gallardo-Cruz JA. 2012. Diversidad, amenazas y oportunidades para la conservación del bosque tropical caducifolio en el estado de Oaxaca, México. Ecosistemas 21: 85-100.

Méndez-Toribio M, Martínez-Cruz J, Cortés-Flores J, Rendón-Sandoval FJ, Ibarra-Manríquez G. 2014. Composición, estructura y diversidad de la comunidad arbórea del bosque tropical caducifolio en Tziritzícuaro, Depresión del Balsas, Michoacán, México. Re- vista Mexicana de Biodiversidad 85: 1117-1128. DOI: https://doi.org/10.7550/rmb.43457

Milles L, Newton AC, DeFries RS, Ravilious C, May I, Blyth S, Kapos V, Gordon JE. 2006. A global overview of the conservation status of tropical dry forests. Journal of Biogeography 33: 491-505. DOI: https://doi. org/10.1111/j.1365-2699.2005.01424.x

Miranda F, Hernández-X E.1963. Los tipos de vegetación de México y su clasificación. Boletín de la Sociedad Botánica de México 28: 29-179. DOI: https://doi. org/10.17129/botsci.1084

Oksanen AJ, Blanchet FG, Friendly M, Kindt R, Legendre P, Mcglinn D, Peter M, O’Hara RB, Simpson G, Solymos P, Stevens MH, Szoecs E, Wagner H. 2019. Package "Vegan". Community Ecology Package. https://github. com/vegandevs/vegan (Accessed April 20, 2020).

Ordoñez MJ, Flores O. 1995. Áreas Naturales Protegidas de México. Pronatura. México. https://www.academia. edu/21877456/Áreas_naturales_protegidas_de_México (Accessed, June 4, 2020).

Palacios-Wassenaar OM, Castillo-Campos G, VázquezTorres SM, Medina-Abreo ME. 2018. Estructura y diversidad de plantas leñosas de la selva mediana subcaducifolia en el centro de Veracruz, México. Acta Botanica Mexicana 124: 85-104. DOI: https://doi. org/10.21829/abm124.2018.1279

Pennington R, Prado D, Pendry C. 2000. Neotropical Seasonally Dry Forests and Quaternary vegetation changes. Journal of Biogeography 27: 261-273. DOI: https:// doi.org/10.1046/j.1365-2699.2000.00397.x

Pennington RT, Lewis GP, Ratter J. 2006. Neotropical savanas and seasonally dry forests: plant diversity, biogeography and conservation. Boca Raton: CRC. ISBN: 0849329876

Pérez-García EA, Meave JA, Gallardo C. 2001. Vegetación y flora de la región de Nizanda, istmo de Tehuantepec, Oaxaca, México. Acta Botanica Mexicana 56: 19-88. DOI: https://doi.org/10.21829/abm56.2001.879

Pérez-García EA, Meave JA, Cevallos-Ferríz SR. 2012. Flora and vegetation of the seasonally dry tropics in Mexico: Origin and biogeographical implications. Acta Botanica Mexicana 100: 149-193. DOI: https://doi. org/10.21829/abm100.2012.35

Pineda-García F, Arredondo-Amezcua L, Ibarra-Manríquez G. 2007. Riqueza y diversidad de especies leñosas del bosque tropical caducifolio El Tarimo, Cuenca del Balsas, Guerrero. Revista Mexicana de Biodiversidad 78: 129-139. DOI: https://doi.org/10.22201/ ib.20078706e.2007.001.396

Portillo-Quintero C, Sanchez-Azofeifa GA. 2010. Extent and conservation of tropical dry forests in the Americas. Biological Conservation 143: 144-155. DOI: https://doi.org/10.1016/j.biocon.2009.09.020

Puig H. 1991. Vegetación de la Huasteca (México), estu- 
dio fitogeográfico y ecológico. México, DF: Instituto de Ecología AC, Institut Francais de Recherche Scientifique Pour le Developpement en Cooperation, entre de Etudes Mexicaines et Centramericaines. ISBN: 9686029-12-2

Ramírez-Albores JE. 2007. Avifauna de cuatro comunidades del oeste de Jalisco, México. Revista Mexicana de Biodiversidad 78: 439-457. DOI: http://dx.doi. org/10.22201/ib.20078706e.2007.002.403

RAN [Registro Agrario Nacional]. 2020. Secretaría de desarrollo Agrario, Territorial y Urbano. México. https:// www.gob.mx/ran (Accessed October 4, 2020).

R Core Team. 2020. R, a language and environment for statistical computing. R Foundation for Statistical Computing, Vienna. https://www.R-project.org/ (Accessed April 20, 2020).

Reyes-Hernández H, Aguilar-Robledo M, Aguirre-Rivera JR, Trejo-Vázquez I. 2006. Cambios en la cubierta vegetal y uso del suelo en el área del proyecto Pujal-Coy, San Luis Potosí, México, 1973-2000. Investigaciones Geográficas 59: 26-42. DOI: https://doi.org/10.14350/ rig.30019

Reyes-Hernández H, Galarza RE, Vázquez VB. 2018. Dinámica de los cambios en la cubierta vegetal y usos de la tierra 1996-2016. In: Reyes-Hernández H, DeNova JA y Durán FA, eds. Reserva de la Biosfera Sierra del Abra Tanchipa. Biodiversidad y Acciones para su Conservación. San Luis Potosí, México: Universidad Autónoma de San Luis Potosí-Comisión Nacional de Áreas Natutales Protegidas-Fondo Mexicano para la Conservación de la Naturaleza-Universidad Autónoma de Tamaulipas, pp. 59-78. ISBN: 978-607535-054-7

Rubio-Méndez G, Castillo-Gómez HA, Hernández-Sandoval L, Espinosa-Reyes G, De-Nova JA. 2018. Chronic disturbance affects the demography and population structure of Beaucarnea inermis, a threatened species endemic to Mexico. Tropical Conservation Science 11: 1-12. DOI: https://doi.org/10.1177/1940082918779802

Rzedowski J. 1978. Vegetación de México. México DF: Limusa. ISBN: 968-18-0002-8

Rzedowski J. 1991. Diversidad y orígenes de la flora fanerogámica de México. Acta Botanica Mexicana 14: 3-21. DOI: https://doi.org/10.21829/abm14.1991.611

Rzedowski J, Calderón de Rzedowski G. 2013. Datos para la apreciación de la flora fanerogámica del bosque tropical caducifolio de México. Acta Botanica Mexicana 102: 1-23. DOI: https://doi.org/10.21829/ abm102.2013.229

Sahagún-Sánchez FJ, Reyes-Hernández H. 2018. Impactos por cambio de uso de suelo en las áreas naturales protegidas en la región central de la Sierra Madre Oriental, México. CienciaUAT 12: 6-21. DOI: https:// dx.doi.org/10.29059/cienciauat.v12i2.831
Salas-Morales SH, Schibli L, Nava-Zafra A, SaynesVásquez A. 2007. Flora de la costa de Oaxaca, México (2): lista florística comentada del parque nacional Huatulco. Boletín de la Sociedad Botánica de México 81: 101-130. DOI: https://doi.org/10.17129/ botsci. 1769

Santiago-Pérez AL, Jardel-Peláez E, Cuevas-Guzmán R. 2002. Rareza y estado de conservación de especies arbóreas del bosque mesófilo de montaña en la Reserva de la Biosfera Sierra de Manantlán, México. Boletín del Instituto de Botánica de la Universidad de Guadalajara 10: 5-22.

SARH [Secretaría de Agricultura y Recursos Hidráulicos]. 1987. Memoria de la Comisión de Estudios de la Cuenca Baja del Río Pánuco (1955-1986). México, DF: Secretaría de Agricultura y Recursos Hidráulicos.

Saxena AK, Singh JS. 1984. Tree population structure of certain Himalayan forest associations and implications concerning their future composition. Vegetatio 58: 6169. DOI: https://doi.org/10.1007/BF00044928

SEMARNAT-CONANP [Secretaría del Medio Ambiente y Recursos Naturales-Comisión Nacional de Áreas Naturales Protegidas]. 2016. Prontuario Estadístico y Geográfico de las Áreas Naturales Protegidas de México. México, DF: Secretaría del Medio Ambiente y Recursos Naturales-Comisión Nacional de Áreas Naturales Protegidas https://www.conanp.gob.mx/prontuario/PRONTUARIOANP2016.pdf (Accessed, March $3,2020)$

Shugart HH, West DC. 1980. Forest succession models. BioScience 30: 308-313. DOI: https://doi. org/10.2307/130785

Silva-Aparicio M, Castro-Ramírez AE, Castillo-Campos G, Perales-Rivera H. 2018. Estructura de la vegetación leñosa en tres áreas con Selva Baja Caducifolia en el Istmo-Costa de Oaxaca, México. Revista de Biología Tropical 66: 863-879. DOI: https://dx.doi. org/10.15517/rbt.v66i2.33419

Soler P, Berroteran J, Gil J, Acosta R. 2012. Índice valor de importancia, diversidad y similaridad florística de especies leñosas en tres ecosistemas de los llanos centrales de Venezuela. Agronomía Tropical 62: 25-37.

Sosa V, De-Nova JA. 2012. Endemic angiosperm lineages in Mexico: hotspots for conservation. Acta Botanica Mexicana 100: 293-315. DOI: https://doi. org/10.21829/abm100.2012.38

Sosa V, De-Nova JA, Vázquez-Cruz M. 2018. Evolutionary history of the flora of Mexico: dry forests cradles and museums of endemism. Journal of Systematics and Evolution 56: 523-536. DOI: https://doi.org/10.1111/ jse. 12416

Squeo FA, Cavieres LA, Arancio G, Novoa JE, Matthei O, Marticorena C, Rodríguez R, Arroyo TKM, Muñoz M. 
1998. Biodiversidad de la flora vascular en la región de Antofagasta, Chile. Revista Chilena de Historia Natural 71: 571-591.

Trejo I. 2005. Análisis de la diversidad de la selva baja caducifolia en México. In: Halffter G, Soberón J, Koleff P, Melic A, eds, Sobre Diversidad Biológica: El Significado de las Diversidades Alfa, Beta y Gamma. Zaragoza, España: Sociedad Entomológica Aragonesa, Monografias Tercer Milenio. pp.111-122. ISBN: 84932807-7-1

Trejo I. 2010. Las selvas secas del Pacífico mexicano. In: Ceballos G, Martínez L, García A, Espinosa E, Bezaury-Creel J, Drizo R. Diversidad, amenazas y áreas prioritarias para la conservación de las selvas secas del Pacífico de México. México, DF: Comisión Nacional para el Conocimiento y Uso de la Biodiversidad, pp. 41-51. ISBN 970-9000-38-1

Trejo I, Dirzo R. 2002. Floristic diversity of Mexican seasonally dry tropical forests. Biodiversity and Conservation 11: 2063-2048. DOI: https://doi. org/10.1023/A:1020876316013

Ugalde AL. 1981. Conceptos básicos de dasometría. Centro Agronómico Tropical de Investigación y Enseñanza (CATIE), Programa de Recursos Naturales Renovables, Turrialba, Costa Rica. http://repositorio.bibliotecaorton. catie.ac.cr/bitstream/handle/11554/886/Conceptos basicos de dasometria.pdf? sequence $=1 \&$ is Allowed $=\mathrm{y}$ (Accessed, July 19, 2020).

Valiente-Banuet A, González-Medrano F, Piñero D. 1995. La vegetación selvática de la región de Gómez Farías, Tamaulipas, México. Acta Botanica Mexicana 33: 1-36. DOI: https://doi.org/10.21829/ $\underline{\operatorname{abm} 33.1995 .751}$

Vázquez-Villa BM, Reyes-Hernández H, Leija Loredo EG, Rivera-González JG, Morera-Beita C. 2020. Environmental governance and conservation. Experiences

\footnotetext{
Editor de sección: Hilda Flores Olvera

Contribución de los autores:

RGH diseñó la investigación, realizó el trabajo de campo, la identificación taxonómica, el registro de los datos, su análisis, revisión de la nomenclatura y la estructuración del manuscrito. FJSS participó en la actualización de la información, la escritura y preparación de la versión final del manuscrito. PDS participó en el análisis de datos, en la estructuración y revisión del manuscrito. PCL participó en el trabajo de campo, la identificación taxonómica y en la revisión del manuscrito. JFM participó en el registro de los datos, la identificación taxonómica y la estructuración del manuscrito. HRH participó en la elaboración del material cartográfico y en la revisión del manuscrito. JADN diseñó la investigación, realizó el trabajo de campo, la identificación taxonómica, el registro de los datos, su análisis, revisión de la nomenclatura y la estructuración del manuscrito. Todos los autores han contribuido sustancialmente con ideas y revisiones de las versiones previas del manuscrito.
}

in two natural protected areas of Mexico and Costa Rica. Journal of Land Use Science 15: 1-14. DOI: https://doi.org/10.1080/1747423X.2020.1817167

Vergara-Paternina JA, Ballesteros-Correa J, GonzálezCharrasquiel C, Linares-Arias JC. 2017. Diversidad de aves en fragmentos de bosque tropical estacionalmente seco en paisajes ganaderos del Departamento de Córdoba, Colombia. Revista de Biología Tropical 65: 1625-1634. DOI: http://dx.doi.org/10.15517/rbt. v65i4.26313

Villaseñor JL. 2003. Diversidad y distribución de las Magnoliophyta de México. Interciencia 28: 160-167.

Villaseñor JL. 2016. Checklist of the native vascular plants of Mexico. Revista Mexicana de Biodiversidad 87: 559-902. DOI: https://doi.org/10.1016/j. $\underline{\mathrm{rmb} .2016 .06 .017}$

Ward Jr JH. 1963. Hierarchical grouping to optimize an objective function. Journal of the American Statistical Association 58: 236-244. DOI: https://doi.org/10.1080/ $\underline{01621459.1963 .10500845}$

Wendt T. 1993. Composition, floristic affinities, and origins of the canopy tree flora of the Mexican Atlantic slope rain forest. In: Ramamoorthy TP, R Bye, A Lot, $\mathrm{J}$ Fa, eds. Biological diversity of Mexico: Origins and Distribution. New York: Oxford University Press, pp. 595-680. ISBN: 019506674X

Williams-Linera G, Lorea F. 2009. Tree species diversity driven by environmental and anthropogenic factors in tropical dry forest fragments of central Veracruz, Mexico. Biodiversity and Conservation 18: 3269-3293. DOI: https://doi.org/10.1007/s10531-009-9641-3

Zamora-Crescencio P, Gutiérrez-Báez C, Villegas P, Domínguez-Carrasco MR, Barrientos-Medina RC. 2016. Composición y estructura del componente arbóreo de la vegetación secundaria en Campeche, México. Foresta Veracruzana 18: 17-24. 